\title{
Regarding the Use of Tamoxifen Post-0ophorectomy to Prevent Hereditary Breast Cancer
}

\author{
Steven A Narod \\ Centre for Research on Women's Health, 790 Bay Street, Toronto, Ontario, Canada M5G 1N8, phone 41635137 65, Email: steven.narod@wchospital.ca
}

Submitted: 5 September 2006

Accepted: 5 December 2006

Options for the prevention of breast cancer in carriers of a BRCA1 or BRCA2 mutation include oophorectomy and tamoxifen [1]. It is possible to reduce the risk of breast cancer by $60 \%$ by performing surgical menopause at age 40 or before [2]; however, given the projected lifetime risk of $80 \%$ and penetrance of $30 \%$ by age 40 , this leaves a residual risk post-oophorectomy of $30 \%$. What can be done to reduce this risk? A healthy diet should be recommended [3] but surely it is optimistic to assume that risk can be managed by diet alone. There is interest in the potential use of aromatase inhibitors in chemoprevention, but none have yet been approved in this setting, and their side effects have not yet been fully evaluated. Raloxifene and tamoxifen appear to have equivalent effects in chemoprevention in women at average or medium risk [4], but raloxifene has not been studied in mutation carriers. Tamoxifen has been shown to reduce the risk of contralateral and ipsilateral breast cancer in several studies of BRCA1 and BRCA2 carriers [5-8]. Few would dispute that oophorectomy should be recommended by age 40 to reduce the risk of breast and ovarian cancer, but there is no consensus on the use of tamoxifen - and its use is not widespread. There are some data on the joint effect of tamoxifen and oophorectomy in women with BRCA1 and BRCA2 mutations - this is all based on evaluating the risks of contralateral breast cancer. Unfortunately, we should not expect data on primary prevention with tamoxifen any time soon. Therefore, it is important to examine the published data on contralateral risk.

Metcalfe et al. conducted a historical cohort study on 491 women with a BRCA1 or BRCA2 mutation who were followed for contralateral breast cancer [6]. The combination of tamoxifen and oophorectomy was particularly effective in women under 50; the hazard ratio associated with this combination of therapy was 0.09 (95\% Cl, 0.01 to $0.68 ; P=0.02)$.
In a matched case-control study, Gronwald et al. studied 285 women with bilateral breast cancer and a BRCA1 or BRCA2 mutation, and 751 control women with unilateral breast cancer and a BRCA1 or BRCA2 mutation [7]. The use of tamoxifen for treating the first breast cancer was compared between bilateral and unilateral cases. The multivariate odds ratio for contralateral breast cancer associated with tamoxifen use was 0.50 for carriers of BRCA 1 mutations and was 0.42 for carriers of BRCA2 mutations. However, in contrast to Metcalfe's study, the protective effect of tamoxifen was not present among women who had undergone an oophorectomy $(O R=0.83)$. However, this subgroup was small and the confidence interval was wide $195 \% \mathrm{Cl}$, 0.24-2.89). A strong protective effect of tamoxifen was apparent among women who had undergone natural menopause $(O R=0.44 ; 95 \% \mathrm{Cl}, 0.27-0.65)$.

Should tamoxifen be given to women with mutations who have had an oophorectomy? Clearly, more data are needed. In women over 50, or who had an oophorectomy after natural menopause, tamoxifen should be effective. In young women $(<50)$ following surgical menopause, the data are less clear-cut, but I think the drug should be considered. Factors to take into consideration when making this decision should include the estimate of the residual lifetime risk of cancer, the woman's level of interest in mastectomy (there is no point in giving tamoxifen to healthy women after prophylactic mastectomy), the presence of an intact uterus (i.e., risk of endometrial cancer) and access to MRI screening facilities.

\section{References}

1. Narod SA, Offit K. Prevention and management of hereditary breast cancer. J Clin Oncol 2005; 23: 1656-1663. 
2. Eisen A, Lubinski J, Klijn J, Moller P, Lynch HT, Offit K, Weber B, Rebbeck T, Neuhausen SL, Ghadirian P, Foulkes WD, Gershoni-Baruch $R$, Friedman E, Rennert G, Wagner T, Isaacs C, Kim-Sing C, Ainsworth P, Sun P, Narod SA. Breast cancer risk following bilateral oophorectomy in BRCA1 and BRCA2 mutation carriers: an international case-control study. J Clin Oncol 2005; 23: 7491-7496.

3. Kotsopoulos J, Narod SA. Towards a dietary prevention of hereditary breast cancer. Cancer Causes Control 2005; 16: 125-138.

4. Vogel VG, Costantino JP, Wickerham DL, Cronin WM, Cecchini RS, Atkins JN, Bevers TB, Fehrenbacher L, Pajon ER Jr, Wade JL 3rd, Robidoux A, Margolese RG, James J, Lippman SM, Runowicz CD, Ganz PA, Reis SE, McCaskill-Stevens W, Ford LG, Jordan VC, Wolmark N; National Surgical Adjuvant Breast and Bowel Project (NSABP). Effects of tamoxifen vs raloxifene on the risk of developing invasive breast cancer and other disease outcomes: the NSABP Study of Tamoxifen and Raloxifene (STAR) P-2 trial. JAMA 2006; 295: 2727-2741.

5. Narod SA, Brunet JS, Ghadirian P, Robson M, Heimdal K, Neuhausen SL, Stoppa-Lyonnet D, Lerman C, Pasini B, de los Rios P, Weber B, Lynch H; Hereditary Breast Cancer Clinical Study Group. Tamoxifen and risk of contralateral breast cancer in BRCA1 and BRCA2 mutation carriers: a case-control study. Lancet 2000; 356:1876-1881.

6. Metcalfe K, Lynch HT, Ghadirian P, Tung N, Olivotto I, Warner E, Olopade OI, Eisen A, Weber B, McLennan J, Sun P, Foulkes WD, Narod SA. Contralateral breast cancer in BRCA1 and BRCA2 mutation carriers. J Clin Oncol 2004; 22: 2328-2335.

7. Gronwald J, Tung N, Foulkes WD, Offit K, Gershoni R, Daly M, Kim-Sing C, Olsson H, Ainsworth P, Eisen A, Saal H, Friedman E, Olopade O, Osborne M, Weitzel J, Lynch H, Ghadirian P, Lubinski J, Sun P, Narod SA; Hereditary Breast Cancer Clinical Study Group. Tamoxifen and contralateral breast cancer in BRCAl and BRCA2 carriers: an update. Int J Cancer 2006; 1 18: 2281-2284.

8. Pierce LJ, Levin AM, Rebbeck TR, Ben-David MA, Friedman E, Solin LJ, Harris EE, Gaffney DK, Haffty BG, Dawson LA, Narod SA, Olivotto IA, Eisen A, Whelan TJ, Olopade OI, Isaacs C, Merajver SD, Wong JS, Garber JE, Weber BL. Ten-year multiinstitutional results of breast-conserving surgery and radiotherapy in BRCA1/2-associated stage I/II breast cancer. J Clin Oncol 2006; 24: 2437-2443. 